CORRECTION

https://doi.org/10.1038/s41586-018-0506-3

\title{
Publisher Correction: Suppression of insulin feedback enhances the efficacy of PI3K inhibitors
}

Benjamin D. Hopkins, Chantal Pauli, Xing Du,

Diana G. Wang, Xiang Li, David Wu, Solomon C. Amadiume, Marcus D. Goncalves, Cindy Hodakoski, Mark R. Lundquist, Rohan Bareja, Yan Ma, Emily M. Harris, Andrea Sboner, Himisha Beltran, Mark A. Rubin, Siddhartha Mukherjee \& Lewis C. Cantley

Correction to: Nature https://doi.org/10.1038/s41586-018-0343-4, published online 04 July 2018.

In this Letter, author Xing Du was incorrectly listed as Du Xing; this has been corrected online. 